\title{
Computation of Coulomb and exchange radial intracule densities
}

\author{
Aaron M. Lee *, Peter M.W. Gill \\ Department of Chemistry, University of Cambridge, Cambridge CB2 1EW, UK
}

Received 30 July 1999; in final form 20 August 1999

\begin{abstract}
The computation of the Coulomb and exchange components $J(u)$ and $K(u)$, respectively, of the Hartree-Fock radial intracule density within the PRISM approach is discussed. Formulae are presented for the even-origin derivatives of these quantities and for the even-order moments of $J(u)$. For molecular systems, we demonstrate that $J(u)$ has, as expected, long-range nature comparable with the molecular extent but, in contrast, that $K(u)$ is relatively short-range, with delocalisation effects providing additional structure and enhancing the range of the intracule. (C) 1999 Elsevier Science B.V. All rights reserved.
\end{abstract}

\section{Introduction}

The evaluation of the Coulomb [1-8] and exchange $[9,10]$ energies with a computational cost that grows linearly with the system size has been an active field of research over the past few years. Algorithms for the construction of the Coulomb energy include hierarchical methods using multipole expansions [2-4], techniques which mathematically partition the Coulomb operator [5,8] and approaches based on the divide-and-conquer philosophy $[6,7,11]$. The existing approaches for the construction of exchange terms $[9,10,12]$ are all based on the exponential decay of the density matrix in insulators [13]. It is clear that insight into the nature of interelectronic interactions is important in the development of efficient algorithms for the computation of the Coulomb and exchange energies. In this Letter, we argue that

\footnotetext{
* Corresponding author. Fax: +44-1223-33-6362; e-mail: aml@ theor.ch.cam.ac.uk
}

construction and analysis of the Coulomb and exchange intracule densities may provide such insight.

The intracule density $I(\boldsymbol{u})$ [14] (Eq. (Eq. (1))) is the probability density for the interelectronic vector $\boldsymbol{u}$,

$I(\boldsymbol{u})=\int \rho_{2}(\boldsymbol{r}, \boldsymbol{r}+\boldsymbol{u}) \mathrm{d} \boldsymbol{r}$,

where $\rho_{2}\left(\boldsymbol{r}_{1}, \boldsymbol{r}_{2}\right)$ is the diagonal second-order spinless density matrix [15]. The spherical integral of this quantity is the radial intracule density,

$P(u)=\int I(\boldsymbol{u}) \mathrm{d} \Omega_{u}$.

(The Jacobian factor $u^{2}$ is included in this integration.) The intracule densities $I(\boldsymbol{u})$ and $P(u)$ have been the focus of many studies and their computation has been considered in a number of papers [16-18]. In recent work, the intracule density of the uniform electron gas [19], the MP2 intracules of a number of atomic systems [18], and the intracule 
Laplacian distribution for the hydrocarbons $\mathrm{C}_{2} \mathrm{H}_{n}$ ( $n=2,4,6)$ [20] have been examined, for example.

In this Letter, we examine intracules derived from Hartree-Fock (HF) wavefunctions and, therefore, consider only HF $\rho(\boldsymbol{r})$ and $\rho_{2}\left(\boldsymbol{r}_{1}, \boldsymbol{r}_{2}\right)$. The HF $P(u)$ can be decomposed into Coulomb and exchange components:

$P(u)=J(u)+K(u)$,

where the radial Coulomb intracule density $J(u)$ is

$J(u)=\frac{1}{2} \iint \rho(\boldsymbol{r}) \rho(\boldsymbol{r}+\boldsymbol{u}) \mathrm{d} \boldsymbol{r} \mathrm{d} \Omega_{u}$.

The Coulomb and exchange intracules $J(u)$ and $K(u)$ integrate to $N^{2} / 2$ and $-N / 2$, respectively, where $N$ is the number of electrons. The Coulomb and exchange energies are given as the integrals

$E_{J}=\int_{0}^{\infty} u^{-1} J(u) \mathrm{d} u$,

$E_{K}=\int_{0}^{\infty} u^{-1} K(u) \mathrm{d} u$,

and it can be shown that $J(u) \geqslant 0$ and $K(u) \leqslant 0$ for all $u . J(u)$ is proportional to the probability density function for the separation $u$ between two electrons moving independently in the density $\rho(\boldsymbol{r})$.

In Section 2, we discuss an efficient approach to the computation of the integrals necessary to assemble radial intracule densities. The computation of moments of the Coulomb intracule is examined in Section 3 and a generalised formulation to compute the even derivatives of the Coulomb and exchange radial intracule densities at the origin is discussed in Section 4. Finally, in Section 5, we present radial intracules for a number of systems. The calculation of radial intracules, derivatives and moments has been implemented in a development version of QCHem [21].

\section{Calculation of radial intracule densities}

In this section, we discuss the computation of the Coulomb and exchange intracules. To compute these intracules, we exploit the fact that $J(u)$ and $K(u)$ can be written as integrals over the 2-electron operator [22]

$$
\frac{f_{u}\left(r_{12}\right)}{r_{12}}=\delta\left(u-r_{12}\right), \quad u>0
$$

where $r_{12}=\left|\boldsymbol{r}_{1}-\boldsymbol{r}_{2}\right|$. Consequently, the intracules can be assembled from the 2-electron integrals

$$
\begin{aligned}
(\boldsymbol{a} \boldsymbol{b} \mid \boldsymbol{c d})_{u}= & \iint \phi_{\boldsymbol{a}}\left(\boldsymbol{r}_{1}\right) \phi_{\boldsymbol{b}}\left(\boldsymbol{r}_{1}\right) \frac{f_{u}\left(r_{12}\right)}{r_{12}} \phi_{\boldsymbol{c}}\left(\boldsymbol{r}_{2}\right) \\
& \times \phi_{\boldsymbol{d}}\left(\boldsymbol{r}_{2}\right) \mathrm{d} \boldsymbol{r}_{1} \mathrm{~d} \boldsymbol{r}_{2},
\end{aligned}
$$

where $\phi_{a}(\boldsymbol{r})$ is a contracted Gaussian-type orbital, by contraction with the density matrix in a manner analogous to the formation of the Coulomb and exchange energies. In the PRISM [23,24] approach, the $(\boldsymbol{a} \boldsymbol{b} \mid \boldsymbol{c d})_{u}$ are constructed from the fundamental integrals $[m]_{u}$. The step that will be discussed here is the formation of the integrals $[\mathrm{m}]_{u}$ of the radial intracule operator (7).

For a general multiplicative 2-electron operator of a scalar argument, $f\left(r_{12}\right) / r_{12}$, the [0] integrals may be computed in real-space using the expression [25]

$$
\begin{aligned}
{[0]=} & U_{P} U_{Q} \frac{2 \theta \exp (-T)}{\sqrt{\pi T}} \\
& \times \int_{0}^{\infty} f\left(\frac{s}{\theta}\right) \exp \left(-s^{2}\right) \sinh (2 s \sqrt{T}) \mathrm{d} s .
\end{aligned}
$$

$T, \theta, U_{P}$ and $U_{Q}$ have their usual meanings [23]. Algebraic forms for the $[m]$ integrals are obtained by differentiating the $[0]$ :

$[m]=(-2)^{m} \theta^{2 m}\left(\frac{\partial^{m}[0]}{\partial T^{m}}\right)_{\theta}$,

considering the [0] a function of the shell-quartet quantities $T$ and $\theta$.

For integrals over the radial-intracule operator (7), one obtains

$$
\begin{aligned}
{[0]_{u}=} & U_{P} U_{Q} \frac{2 u \theta^{2} \exp (-T)}{\sqrt{\pi T}} \exp \left(-u^{2} \theta^{2}\right) \\
& \times \sinh (2 u \theta \sqrt{T}), \quad u>0 .
\end{aligned}
$$

The higher-order fundamental integrals $[\mathrm{m}]_{u}$ are most compactly written in terms of the functions $q_{n}(x)$,

$q_{n}(x)=\frac{\exp (-x)}{x^{n}} i_{n}(x)$, 
where the $i_{n}(x)$ are modified spherical Bessel functions of the first kind [26],

$i_{n}(x)=x^{n}\left(\frac{1}{x} \frac{\mathrm{d}}{\mathrm{d} x}\right)^{n} \quad \frac{\sinh (x)}{x}$.

The $q_{n}(x)$ are well-behaved for small and large $x$ and decrease monotonically from the origin. The functions have the limits

$$
\begin{aligned}
& \lim _{x \rightarrow 0} q_{n}(x)=\frac{1}{(2 n+1) ! !}, \\
& \lim _{x \rightarrow \infty} q_{n}(x)=0 .
\end{aligned}
$$

Using the $q_{n}(x)$, one finds

$$
\begin{aligned}
{[m]_{u}=} & U_{P} U_{Q} \frac{2 u^{2} \theta\left(2 \theta^{2}\right)^{m+1}}{\sqrt{\pi}} \exp \left[-(u \theta-\sqrt{T})^{2}\right] \\
& \times \sum_{k=0}^{m}\left(\begin{array}{c}
m \\
k
\end{array}\right)(-2)^{k}(u \theta)^{2 k} q_{k}(2 u \theta \sqrt{T}),
\end{aligned}
$$

for $u>0$. We will later consider the right-hand derivatives of the $[\mathrm{m}]_{u}$ with respect to $u$.

To speed up the computation of the $q_{n}(x)$, we use interpolation tables [27] for $x<x_{\max }$ and asymptotic expansions outside this range $\left(x_{\max }\right.$ is chosen such that the error in the use of asymptotic expansions is negligible, for example $x_{\max }=1000$ ). The asymptotic expansion of $I_{\nu}(x)$ [26] may be used to derive the expansion for the $q_{n}(x)$,

$$
\begin{aligned}
q_{n}(x) \sim & \frac{1}{2 x^{n+1}}\left[1-\frac{(\mu-1)}{(8 x)}+\frac{(\mu-1)(\mu-9)}{2 !(8 x)^{2}}\right. \\
& \left.-\frac{(\mu-1)(\mu-9)(\mu-25)}{3 !(8 x)^{3}}+\cdots\right],
\end{aligned}
$$

where $\mu=(2 n+1)^{2}$ and $|x| \rightarrow \infty$. These asymptotic expansions terminate when $n$ is an integer, as is the case in (16). In the present approach, we have used a cubic Taylor interpolation table for $q_{n}(x), n=$ $1,2, \ldots n_{\max }$. The table may be evaluated once the accuracy and cutoff $x_{\max }$ have been specified.

When computing a radial intracule density for a range of points, it is clear that shell-quartet quantities such as $T$ and $\theta$ should not be recomputed for each point, as observed by Cioslowski and Liu [17]. In the present implementation, the loop over points has been located within the loop over shell-quartets and this approach has been further refined by splitting the points loop into two regions: the first where interpolation is used for the $q_{n}$, and the second where asymptotic expansions are used. This strategy maximises savings gained using interpolation. For a grid of equally separated points, it is straightforward to determine the index of the division point.

\section{Computation of radial intracule moments}

The $s$-order moments of the Coulomb and exchange intracules

$J_{s}=\int_{0}^{\infty} u^{s} J(u) \mathrm{d} u$,

$K_{s}=\int_{0}^{\infty} u^{s} K(u) \mathrm{d} u$,

can, in general, be constructed from the fundamental 2-electron integrals $[0]_{s}$ of the operator

$\frac{f_{s}\left(r_{12}\right)}{r_{12}}=r_{12}^{s}$,

using (9) and the auxiliary integrals $[\mathrm{m}]_{s}$ then determined via (10). In principle, these expressions can be evaluated for all $s>-3$. We do not investigate this approach at the present time.

For $s$ an even integer $2 n$, the moments of the Coulomb radial intracule can be formed more efficiently by taking advantage of the factorisation of $r_{12}^{2 n}$ to yield [28]

$$
\begin{aligned}
J_{2 n}= & \frac{1}{2} \sum_{p=0}^{n}(-2)^{p} \sum_{q=0}^{n-p} f_{n p q}\left\langle r^{2(n-p-q)}\left(r_{\alpha} r_{\beta} \cdots\right)_{p}\right\rangle \\
& \times\left\langle r^{2 q}\left(r_{\alpha} r_{\beta} \cdots\right)_{p}\right\rangle .
\end{aligned}
$$

This expression can be reformulated as

$J_{2 n}=\frac{1}{2} \sum_{p=0}^{n}(-2)^{p} \sum_{q=0}^{n-p} f_{n p q} Z_{n p q}$, 
where

$f_{k p q}=\frac{k !}{p ! q !(k-p-q) !}$,

$Z_{k p q}=\sum_{n=0}^{p} \sum_{m=0}^{p-n} f_{p n m} T_{p n m}^{(k-p-q)} T_{p n m}^{(q)}$,

$T_{p n m}^{(k)}=\int r^{2 k} x^{n} y^{m} z^{(p-n-m)} \rho(\boldsymbol{r}) \mathrm{d} \boldsymbol{r}$.

This formulation allows the computation of the $J_{2 n}$ for arbitrary integral $n \geqslant 0$ with a cost that grows linearly with the system size. The computation of the $J_{2 n}$ in Q-CHEM [21] was first performed by Adamson [29] using explicit formulae for a range of $n$ values.

\section{Computation of radial intracule derivatives}

While the moments of the radial intracule density $P(u)$ have been thoroughly studied, both for correlated and uncorrelated wavefunctions, its derivatives from the right have not been investigated in detail. In Section 4, we examine the computation of derivatives of the radial intracule density. The expansion of the radial intracule density about $u=0^{+}$may be written as

$P(u)=P^{(2)} u^{2}+P^{(3)} u^{3}+\cdots$,

where

$\left.P^{(n)} \equiv \frac{1}{n !}\left(\frac{\partial^{n} P(u)}{\partial u^{n}}\right)\right|_{u=0^{+}}$.

If all of the derivatives of the density $\rho(\boldsymbol{r})$ and density matrix $\rho_{2}\left(\boldsymbol{r}_{1}, \boldsymbol{r}_{2}\right)$ exist at all points in space (which is true for HF calculations using Gaussian basis functions) then the expansions of $P(u)$ and $J(u)$ contain only even terms and therefore

$P^{(2 n+2)}=\frac{4 \pi}{(2 n+1) !} \int\left[\left(\nabla_{\boldsymbol{r}^{\prime}}^{2}\right)^{n} \rho_{2}\left(\boldsymbol{r}, \boldsymbol{r}^{\prime}\right)\right]_{\boldsymbol{r}^{\prime}=\boldsymbol{r}} \mathrm{d} \boldsymbol{r}$,

$J^{(2 n+2)}=\frac{2 \pi}{(2 n+1) !} \int \rho(\boldsymbol{r})\left(\nabla_{r}^{2}\right)^{n} \rho(\boldsymbol{r}) \mathrm{d} r$,

providing an interesting route to the computation of these quantities. $K^{(2 n+2)}$ is defined as the difference $P^{(2 n+2)}-J^{(2 n+2)}$. Using integration-by-parts, it is straightforward to prove that $J^{(2 n)}$ and $K^{(2 n)}$ alternate in sign as $n$ increments. It may also be shown that $J^{(2)}>0$ and $K^{(2)}<0$ and, for closed-shell systems, that $J^{(2)}=-2 K^{(2)}$.

Differentiation of (11) with respect to $u$ and evaluation at $u=0$,

$\left.[0]^{(2 n)} \equiv \frac{1}{(2 n) !}\left(\frac{\partial^{2 n}[0]_{u}}{\partial u^{2 n}}\right)\right|_{u=0^{+}}$,

leads immediately to the fundamental integrals for the even intracule derivatives. Expressed in terms of the Hermite polynomials $H_{n}(x)$ [26], these are

$$
\begin{aligned}
{[0]^{(2 n)}=} & U_{P} U_{Q} \frac{2 \theta^{2 n+1}}{\sqrt{\pi T}(2 n-1) !} \\
& \times \exp (-T) H_{2 n-1}(\sqrt{T}), \quad n=1,2, \ldots
\end{aligned}
$$

with $[0]^{(0)}=0$. Using the recurrence properties of the Hermite polynomials, it follows that

$$
\begin{aligned}
{[0]^{(2 n+2)}=} & \frac{-2 \theta^{2}}{n(2 n+1)}\left[(2 n-T-1 / 2)[0]^{(2 n)}\right. \\
& \left.+\theta^{2}[0]^{(2 n-2)}\right], \quad n=1,2, \ldots
\end{aligned}
$$

A two-index recurrence relationship for higher-order fundamental integrals can be derived by using Leibniz' formula to apply the definition of (10) to (32)

$$
\begin{aligned}
{[m]^{(2 n+2)}=} & \frac{-2 \theta^{2}}{n(2 n+1)}\left[(2 n-T-1 / 2)[m]^{(2 n)}\right. \\
& \left.+2 \theta^{2} m[m-1]^{(2 n)}+\theta^{2}[m]^{(2 n-2)}\right], \\
n=1,2, \ldots &
\end{aligned}
$$

The boundary conditions for this recurrence are

$[m]^{(0)}=0$,

$[m]^{(2)}=U_{P} U_{Q} \frac{2 \theta\left(2 \theta^{2}\right)^{m+1}}{\sqrt{\pi}} \exp (-T)$,

$$
m=0,1, \ldots .
$$

We note in passing that the use of interpolation tables for quantities such as

$$
\frac{\exp (-T) H_{2 n-1}(\sqrt{T})}{\sqrt{T}(2 n-1) !}
$$


and its derivatives with respect to $T$ should yield an improvement in efficiency over a recursive evaluation.

\section{Results and discussion}

In Section 5, we examine Coulomb and exchange intracules computed using unrestricted HF wavefunctions. To test our intracule curves, we have verified that we obtain, by numerical integration, the correct normalisation integrals, and energies for a number of test systems. The moments of the $J(u)$ are consistent with those obtained by numerical integration. Satisfactory agreement between the lower-order derivatives of $J(u)$ and $K(u)$ computed numerically and those computed analytically for a number of diatomic molecules has also been obtained. We have reproduced the Coulomb + exchange energy, normalisation integral, first- and second-order moments of $P(u)$ given by Wang et al. [30] for $\mathrm{Ne}, \mathrm{H}_{2}$ and LiH. With the exception of the peptide endothelin-1 [31], the intracule curves plotted have been computed using a 0.1 bohr point separation and high densitymatrix convergence, integral thresholds and interpolation table accuracy. For endothelin-1, a 0.05 bohr point separation was used along with lower values for the convergence, thresholds and accuracy parameters. All calculations use a value of $x_{\max }=1000$ for the interpolation table cutoff. The plotted curves have been interpolated [32]. All results are given in atomic units, e.g., $u$ is in bohr and $P(u), J(u)$ and $K(u)$ are in hartree.
In Table 1, the base-2 logarithms of the quantities $\left|J^{(2 n)}\right|,\left|K^{(2 n)}\right|$ and $J_{2 n}$ are given for a sequence of all-trans polyalkenes $\left(r_{\mathrm{C}-\mathrm{C}}=1.46 \AA, r_{\mathrm{C}=\mathrm{C}}=1.32\right.$ $\AA, r_{\mathrm{CH}}=1.09 \AA$, trigonal angles at each $\mathrm{C}$ atom) with the STO-3G basis set. For each polyalkene, the growth of the $J^{(2 n)}$ is approximately exponential, $\left|J^{(2 n+2)}\right| \approx 8\left|J^{(2 n)}\right|$. Each polyalkene in the sequence is approximately double the preceding member in size and, for fixed $2 n$, the $J^{(2 n)}$ almost exactly double moving up the sequence, reflecting that $J^{(2 n)}$ is a linear scaling property. The base- 2 logarithms of the $\left|K^{(2 n)}\right|$ behave similarly to those of the $\left|J^{(2 n)}\right|$. The results illustrate the relationship $J^{(2)}=-2 K^{(2)}$. For each polyalkene, the growth of the $J_{2 n}$ is also approximately exponential, although the factor of increase is not constant over the polyalkene series. Rough scaling arguments suggest that the value of $J_{2 n}$ will increase by $2^{2 n}$ moving to an polyalkene double in size and this is also approximately observed. For example, $J_{20}$ is approximately $2^{20}$ times larger in $\mathrm{C}_{8} \mathrm{H}_{10}$ than in $\mathrm{C}_{4} \mathrm{H}_{6}$.

The radial intracule densities $J(u)$ and $K(u)$ of the nitrogen atom and molecule $\left(r_{\mathrm{NN}}=1.12998 \AA\right)$ with the STO-3G and cc-pVQZ basis sets are given in Fig. 1. Thakkar et al. [33] examined the topological features of a $\mathrm{HF} P(u)$ for $\mathrm{N}_{2}$. The features of $J(u)$ are dominated by pairwise interactions between regions of high density. The innermost peak on the curve for the $\mathrm{N}$ atom results from the self-interaction of core electrons while the broad second peak presumably arises from the core-valence and valencevalence interactions. Both of these peaks are present in the $\mathrm{N}_{2}$ intracule which also contains a peak at

Table 1

Base-2 logarithms of the $\left|J^{(2 n)}\right|,\left|K^{(2 n)}\right|$ and $J_{2 n}$ values for a sequence of polyalkenes with the STO-3G basis. The signs of $\left|J^{(2 n)}\right|$ and $\left|K^{(2 n)}\right|$ are $(-1)^{n+1}$ and $(-1)^{n}$, respectively. $J_{2 n}>0$

\begin{tabular}{rccccccccccccc}
\hline $2 n$ & $\log _{2}\left|J^{(2 n)}\right|$ & \multicolumn{3}{c}{$\log _{2}\left|K^{(2 n)}\right|$} & \multicolumn{5}{c}{$\log _{2}\left(J_{2 n}\right)$} \\
\cline { 2 - 13 } & $\mathrm{C}_{4} \mathrm{H}_{6}$ & $\mathrm{C}_{8} \mathrm{H}_{10}$ & $\mathrm{C}_{16} \mathrm{H}_{18}$ & $\mathrm{C}_{32} \mathrm{H}_{34}$ & $\mathrm{C}_{4} \mathrm{H}_{6}$ & $\mathrm{C}_{8} \mathrm{H}_{10}$ & $\mathrm{C}_{16} \mathrm{H}_{18}$ & $\mathrm{C}_{32} \mathrm{H}_{34}$ & $\mathrm{C}_{4} \mathrm{H}_{6}$ & $\mathrm{C}_{8} \mathrm{H}_{10}$ & $\mathrm{C}_{16} \mathrm{H}_{18}$ & $\mathrm{C}_{32} \mathrm{H}_{34}$ \\
\hline 2 & 9.60255 & 10.6017 & 11.6012 & 12.6010 & 8.60255 & 9.60166 & 10.6012 & 11.6010 & 13.2375 & 16.7373 & 20.5403 & 24.4630 \\
4 & 13.8477 & 14.8479 & 15.8479 & 16.8480 & 12.8666 & 13.8667 & 14.8668 & 15.8668 & 18.6297 & 23.9038 & 29.6436 & 35.5396 \\
6 & 17.7695 & 18.7697 & 19.7698 & 20.7698 & 16.7745 & 17.7746 & 18.7747 & 19.7748 & 24.5172 & 31.5253 & 39.1788 & 47.0405 \\
8 & 21.5171 & 22.5173 & 23.5174 & 24.5175 & 20.5189 & 21.5191 & 22.5192 & 23.5192 & 30.7144 & 39.3858 & 48.9345 & 58.7584 \\
10 & 25.0736 & 26.0738 & 27.0740 & 28.0740 & 24.0747 & 25.0749 & 26.0750 & 27.0750 & 37.1365 & 47.4002 & 58.8261 & 70.6091 \\
12 & 28.4346 & 29.4348 & 30.4349 & 31.4350 & 27.4353 & 28.4355 & 29.4356 & 30.4357 & 43.7403 & 55.5275 & 68.8110 & 82.5498 \\
14 & 31.6199 & 32.6201 & 33.6202 & 34.6203 & 30.6204 & 31.6206 & 32.6207 & 33.6208 & 50.5007 & 63.7451 & 78.8647 & 94.5556 \\
16 & 34.6545 & 35.6548 & 36.6549 & 37.6549 & 33.6549 & 34.6551 & 35.6552 & 36.6553 & 57.4015 & 72.0391 & 88.9718 & 106.611 \\
18 & 37.5592 & 38.5594 & 39.5595 & 40.5596 & 36.5594 & 37.5596 & 38.5597 & 39.5598 & 64.4304 & 80.4008 & 99.1224 & 118.705 \\
20 & 40.3483 & 41.3486 & 42.3487 & 43.3487 & 39.3485 & 40.3487 & 41.3488 & 42.3489 & 71.5780 & 88.8242 & 109.309 & 130.831 \\
\hline
\end{tabular}




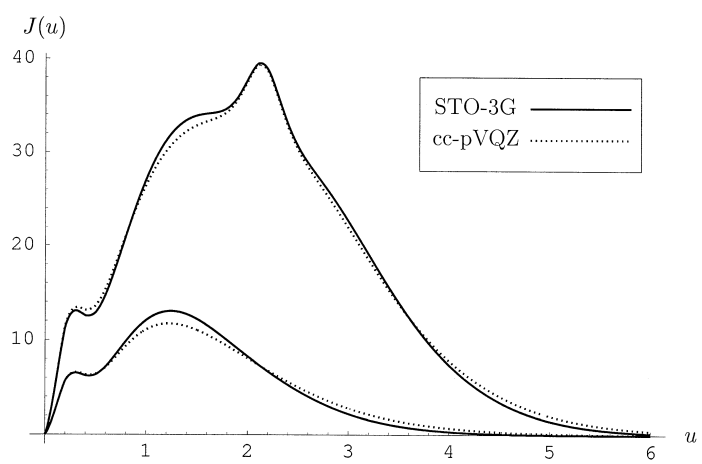

$$
K(u)
$$

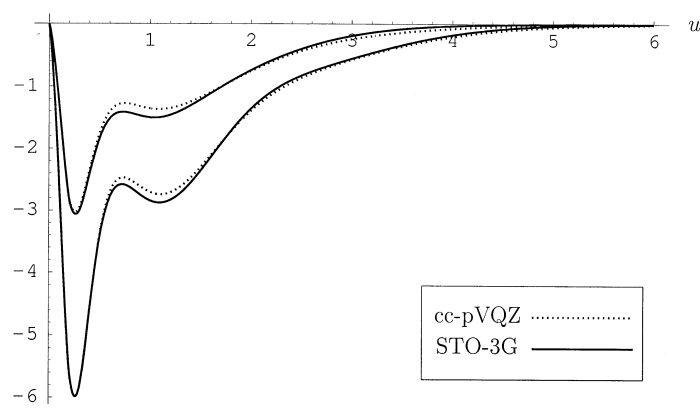

Fig. 1. The intracules $J(u)$ and $K(u)$ for $\mathrm{N}$ (lower curves) and $\mathrm{N}_{2}$ (upper curves) with the STO-3G and cc-pVQZ basis sets.

approximately the internuclear separation primarily resulting from the interaction of core densities on the separate centres. The broad tails of the $\mathrm{N}_{2}$ curves suggests that the internuclear core-valence and valence-valence interactions are buried in this region. Rationalising the effect of exchange as supplying a 'self-interaction' correction, the exchange intracule should have peaks for the core-core and valence-valence self-interactions and this is observed. Since the qualitative features of these intracules remain largely unchanged between the STO-3G and cc-pVQZ basis sets, we have decided to use the small STO-3G basis for our calculations on larger systems.

In Fig. 2, the intracule densities for the polyalkene $\mathrm{C}_{8} \mathrm{H}_{10}$ are given. The maxima in $J(u)$ occur at the values of $u$ corresponding to internuclear separations, as expected, with the peak closest to the origin arising primarily from the self-interaction of the core electrons. The self-interaction effect of $K(u)$ is also evident as is the short-range nature of this intracule compared to Coulomb intracule $J(u)$. In Fig. 3 the exchange intracules for the 'linear' alkane $\mathrm{C}_{8} \mathrm{H}_{18}$

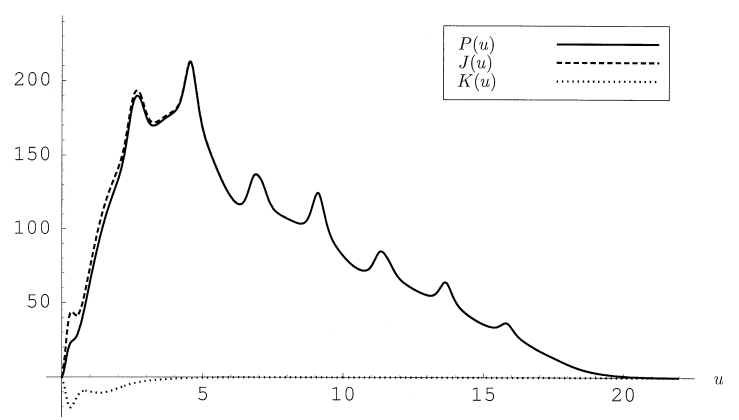

Fig. 2. The intracules $P(u), J(u)$ and $K(u)$ for the polyalkene $\mathrm{C}_{8} \mathrm{H}_{10}$ with the STO-3G basis set.

( $r_{\mathrm{CC}}=1.4 \AA, r_{\mathrm{CH}}=1.09 \AA$, tetrahedral angles at each $\mathrm{C}$ atom) and polyalkene $\mathrm{C}_{8} \mathrm{H}_{10}$ are compared. These intracules are normalised to integrate to -1 . Although the intracules appear very similar near the origin, closer examination shows that oscillations in the tails of the curves are only noticeable for the polyalkene intracule, and we attribute this to delocalisation effects. The total exchange energies are

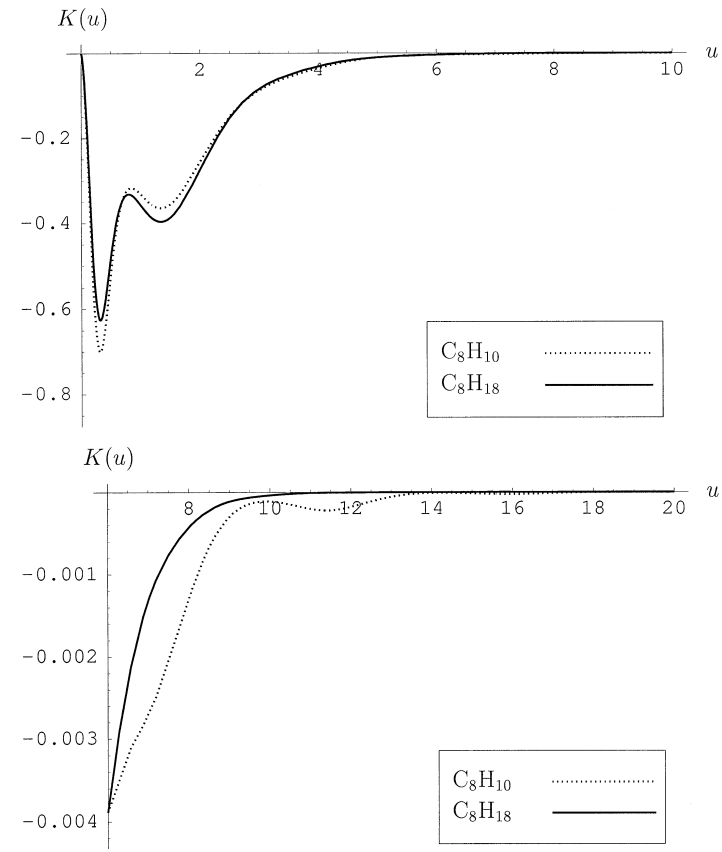

Fig. 3. The exchange intracules for the alkane $\mathrm{C}_{8} \mathrm{H}_{18}$ and polyalkene $\mathrm{C}_{8} \mathrm{H}_{10}$ computed with the STO-3G basis. The curves are normalised to integrate to -1 . 
-48.487 and $-45.099 E_{\mathrm{h}}$ for the alkane and polyalkene, respectively, with exchange energy contributions from $u>6$ bohr of -17 and $-27 m E_{\mathrm{h}}$. Therefore, although the polyalkene has the smaller exchange energy, it contains a larger long-range component.

To explore the effect of delocalisation further, the exchange intracules for the linear hydrogen and helium arrays $\mathrm{H}_{50}$ and $\mathrm{He}_{50}$ (internuclear separation is 1.40 bohr in both) are given in Fig. 4. These intracules have been normalised to integrate to -1 . For the delocalised $\mathrm{H}_{50}$ intracule, the oscillations in the tail region are quite noticeable. In contrast, the $\mathrm{He}_{50}$ exchange intracule is considerably more short-range and lacks the oscillatory signature of delocalisation. The fractions of exchange energy outside $u=4 \mathrm{bohr}$ are $2.0 \%$ and $0.01 \%$ for $\mathrm{H}_{50}$ and $\mathrm{He}_{50}$, respectively, clearly indicative of the longer-range nature of the $\mathrm{H}_{50}$ intracule.

The STO-3G $J(u)$ and $K(u)$ intracules for the peptide endothelin-1 [31] $\left(\mathrm{C}_{82} \mathrm{H}_{121} \mathrm{~N}_{20} \mathrm{O}_{25} \mathrm{~S}_{5}\right)$ are given in Fig. 5. This structure (PDB ID: 1EDP) was

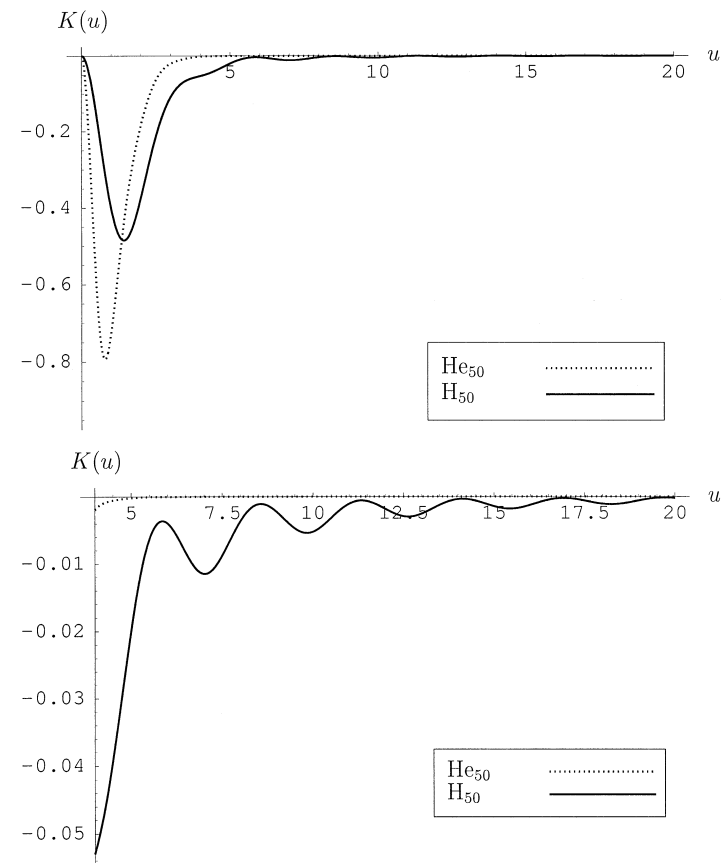

Fig. 4. The exchange intracules for the linear hydrogen and helium arrays $\mathrm{H}_{50}$ and $\mathrm{He}_{50}$ with the STO-3G basis. The curves are normalised to integrate to -1 .
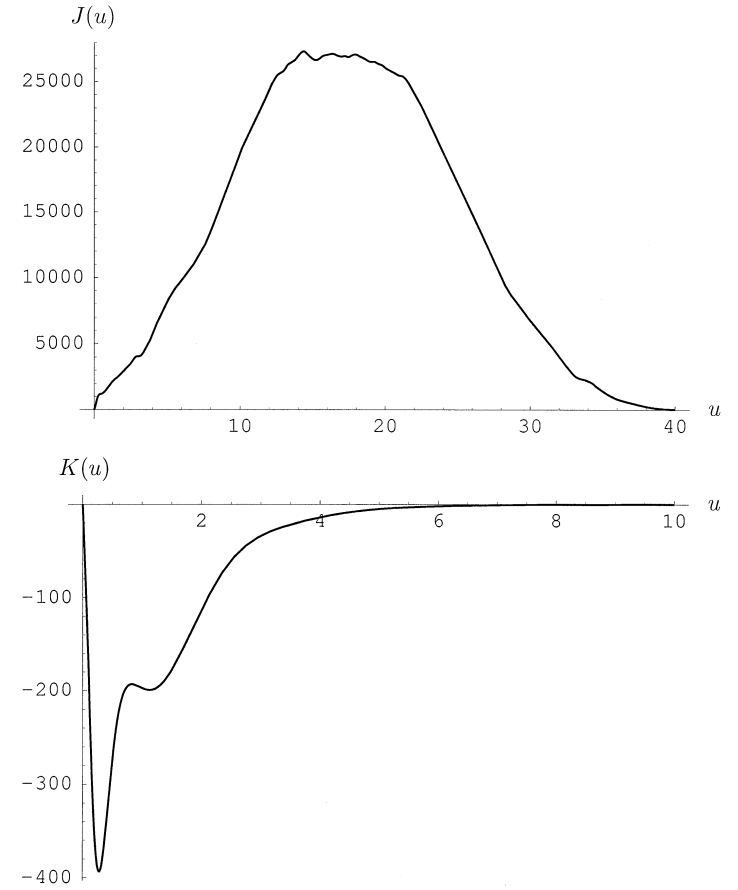

Fig. 5. The intracules $J(u)$ and $K(u)$ for endothelin- 1 with the STO-3G basis set.

obtained from the protein data bank $[34,35]$. We have assumed a charge of +1 for the calculations. The $J(u)$ curve for endothelin-1 differs dramatically from that of $\mathrm{C}_{8} \mathrm{H}_{10}$ in that the internuclear peaks overlap significantly and that it does not contain any 'special distances' resulting from high molecular symmetry. The $K(u)$ curves for these systems, despite the presence of a small number of second-row atoms in endothelin-1, are remarkably similar in the region around the origin.

\section{Conclusions}

In this Letter, we have shown how to compute radial intracule densities and their derivatives using the PRISM approach. The integral expressions presented here should enable the computation of both of these quantities using the PRISM approach for correlated wavefunctions such as CI expansions with Gaussian-type orbitals. Both the Coulomb and exchange intracules $J(u)$ and $K(u)$ yield information 
on electronic structure. $J(u)$ demonstrates the longrange nature of pairwise charge interactions and $K(u)$ shows that exchange effects are usually shortranged with delocalisation introducing distinct structure. Importantly, both $J(u)$ and $K(u)$ show which regions of interelectronic separation $u$ are significant for the computation of, for example, energies. We are currently investigating the possibility of estimating Coulomb and exchange energies directly from moments and derivatives of their intracules and will report our results elsewhere.

\section{Acknowledgements}

This research was supported in part by grants from the US National Science Foundation (SBIR Grant DMI-95314598) and the UK Engineering and Physical Sciences Research Council (Grant GR/M63430). We are also grateful to Q-Chem, Inc., for various forms of assistance and we thank Mr. Nikhil Nair for stimulating discussions.

\section{References}

[1] L. Greengard, Science 265 (1994) 909.

[2] C.A. White, B.G. Johnson, P.M.W. Gill, M. Head-Gordon, Chem. Phys. Lett. 230 (1994) 8.

[3] R. Kutteh, E. Aprá, J. Nichols, Chem. Phys. Lett. 238 (1995) 173.

[4] M.C. Strain, G.E. Scuseria, M.J. Frisch, Science 271 (1996) 51.

[5] J.P. Dombroski, S.W. Taylor, P.M.W. Gill, J. Phys. Chem. 100 (1996) 6272.

[6] R.T. Gallant, A. St.-Amant, Chem. Phys. Lett. 256 (1996) 569.

[7] J.M. Pérez-Jordá, W. Yang, J. Chem. Phys. 107 (1997) 1218.

[8] R.D. Adamson, J.P. Dombroski, P.M.W. Gill, J. Comput. Chem. 20 (1999) 921.

[9] E. Schwegler, M. Challacombe, J. Chem. Phys. 105 (1996) 2726.
[10] J.C. Burant, G.E. Scuseria, M.J. Frisch, J. Chem. Phys. 105 (1996) 8969.

[11] W. Yang, Phys. Rev. Lett. 66 (1991) 1438.

[12] E. Schwegler, M. Challacombe, M. Head-Gordon, J. Chem. Phys. 106 (1997) 9708.

[13] W. Kohn, Int. J. Quantum Chem. 56 (1995) 229.

[14] A.J. Coleman, Int. J. Quantum Chem. Symp. 1 (1967) 457.

[15] R.G. Parr, W. Yang, Density Functional Theory of Atoms and Molecules, Oxford University Press, New York, 1989.

[16] J.M. Ugalde, C. Sarasola, L. Domínguez, J. Math. Chem. 6 (1991) 51.

[17] J. Cioslowski, G. Liu, J. Chem. Phys. 105 (1996) 4151.

[18] J. Cioslowski, G. Liu, J. Chem. Phys. 109 (1998) 8225.

[19] X. Fradera, C. Sarasola, J.M. Ugalde, R.J. Boyd, Chem. Phys. Lett. 304 (1999) 393.

[20] X. Fradera, M. Duran, J. Mestres, J. Chem. Phys. 107 (1997) 3576.

[21] C.A. White, J. Kong, D.R. Maurice, T.R. Adams, J. Baker, M. Challacombe, E. Schwegler, J.P. Dombroski, C. Ochsenfeld, M. Oumi, T.R. Furlani, J. Florian, R.D. Adamson, N. Nair, A.M. Lee, N. Ishikawa, R.L. Graham, A. Warshel, B.G. Johnson, P.M.W. Gill, M. Head-Gordon, QCHEM, Version 1.2, Q-Chem, Inc., Pittsburgh, PA, 1998.

[22] A.J. Thakkar, V.H. Smith Jr., J. Chem. Phys. 67 (1977) 1191.

[23] P.M.W. Gill, Adv. Quantum Chem. 25 (1994) 141.

[24] T.R. Adams, R.D. Adamson, P.M.W. Gill, J. Chem. Phys. 107 (1997) 124

[25] A.M. Lee, Chem. Phys. Lett. 292 (1998) 172.

[26] M. Abramowitz, I.A. Stegun, Handbook of Mathematical Functions, Dover, New York, 1965.

[27] P.M.W. Gill, B.G. Johnson, J.A. Pople, Int. J. Quantum Chem. 40 (1991) 745.

[28] P.M.W. Gill, Chem. Phys. Lett. 270 (1997) 193.

[29] R.D. Adamson, unpublished.

[30] J. Wang, A.N. Tripathi, V.H. Smith Jr., J. Chem. Phys. 97 (1992) 9188.

[31] N.H. Andersen, C. Chen, T.M. Marschner, S.R. Krystek Jr., D.A. Bassolino, Biochemistry 31 (1992) 1280, PDB ID: 1EDP.

[32] S. Wolfram, The Mathematica Book, Wolfram Media/Cambridge University Press, 1996 3rd edn.

[33] A.J. Thakkar, A.N. Tripathi, V.H. Smith Jr., Int. J. Quantum Chem. 26 (1984) 157.

[34] F.C. Bernstein, T.F. Koetzle, G.J. Williams, E.E. Meyer Jr, M.D. Brice, J.R. Rodgers, O. Kennard, T. Shimanouchi, M. Tasumi, J. Mol. Biol. 112 (1977) 535.

[35] The Research Collaboratory for Structural Bioinformatics PDB, http://www.rcsb.org/pdb/ 